Case Reports

\title{
Relapsing Cutaneous Leishmaniasis in a Patient Treated with Humira
}

\author{
${ }^{1}$ Erik Otte, ${ }^{2}$ Mikael Christiansen and ${ }^{1,3}$ Eskild Petersen \\ ${ }^{I}$ Department of Clinical Microbiology, Aarhus University Hospital, Aarhus, Denmark \\ ${ }^{2}$ Department of Rheumatology, Aarhus University Hospital, Aarhus, Denmark \\ ${ }^{3}$ De partment of Infectious Diseases, Aarhus University Hospital, Aarhus, Denmark
}

\author{
Article history \\ Received: 03-12-2014 \\ Revised: 02-01-2015 \\ Accepted: 05-05-2015 \\ Corresponding Author: \\ Eskild Petersen \\ Department of \\ Infectious Diseases and \\ Clinical Microbiology, Aarhus \\ University Hospital, Aarhus, \\ Denmark \\ Email: eskildp@dadlnet.dk
}

\begin{abstract}
Leishmanias is an anthropozoonosis caused by the Leishmania protozoa. The manifestation of the disease varies depending on the type of Leishmania and the immunestatus of the patient. Tumor Necrosis Factor (TNF)- $\alpha$ antagonists were introduced in the late 1990s and have had a marked impact on rheumatic diseases. Tumor necrosis factor plays an important role in the immune systems defense against intracellular infections and the use of TNF- $\alpha$ antagonists is linked with an increased frequency of infections. We here present a case of cutaneous leishmaniasis relapse following treatment with miltefosine and amphotericin $\mathrm{B}$, in a patient undergoing treatment with a TNF- $\alpha$ antagonist.
\end{abstract}

Keywords: Cutaneous Leishmaniasis, Humira, Adalimumab, TNF- $\alpha$ Antagonist, Amphotericin B

\section{Introduction}

Leishmaniasis is an infection caused by the parasite Leishmania, a protozoan zoonosis transmitted by sandflies. Leishmaniasis has several clinical presentations, including a cutaneous, mucosal and a visceral form. Clinical presentation is, to a large extent, determined by the Leishmania subtype of which more than 20 are known. In Central and South America (New World) Cutaneous Leishmaniasis (CL) is mainly caused by $L$. mexicana and L. viannia complexes, with the Lutzomyia sandfly as the primary vector. Old World leishmaniasis (Europe, Africa and Asia) is transmitted by the vector Phlebotomus and is mainly caused by the subtypes L. major, L. tropica and L. aethiopica. Worldwide cutaneous leishmaniasis is the most common presentation of leishmania infection with an estimated annual incidence of 0.7-1.2 million cases (Alvar et al., 2012). CL is characterized by a painless dry noduli or ulceration with indurated borders. The infection can be latent and reactivated when a hosts immune system is compromised. Globally an estimated 350 million people are at risk and a northward spread has been reported with cases of leishmaniasis presenting in northern Italy, central Europe (Aspöck et al., 2008) and the Jura region in France (Faber et al., 2012). Also within the last decade an increasing number of travelers and military personnel returning to Europe, with Leishmania infections, have been reported (Hodiamont et al., 2014). Ankylosing Spondylitis (AS), also known as Morbus
Bechterew, is a chronic inflammatory rheumatic disease that primarily involves the sacroiliacal joints and the spine. The first line treatment is NSAID's and nonpharmaceutical therapy such as exercise and physiotherapy. Within the last decade anti TNF- $\alpha$ therapy has emerged as a strong alternative and is now recommended for patients with persistently high disease activity despite conventional treatment (Braun et al., 2011). Adalimumab (ADA) is a recombinant fully human IgG1 monoclonal antibody which binds with high affinity to soluble TNF- $\alpha$ and blocks its interaction with cell surface $\mathrm{TNF}-\alpha$ receptors. Large randomized placebo-controlled trials with AS patients have proven ADA highly efficient in reducing backpain, loss of function and inflammatory markers (Van der Heijde et al., 2006). Here we report a case of a patient with AS treated with ADA, who presented with a case of relapsing CL after treatment with miltefosine and amphotericin B.

\section{Case Report}

A 62-year old Caucasian man with a 17-year history of AS, uveitis, psoriasis and Crohn's disease, was treated with Asacol, Salazopyrin. ADA was added to the treatment in 2011 with a marked effect on AS related symptoms. In May 2013, while holidaying in Portugal, the patient presented with a cutaneous ulceration on the dorsal side of his left antebrachii. No systemic symptoms were present and initial treatment 
consisted of an oral antibiotic with no significant response. Upon his return to Denmark in July, the patient was seen at the Department of Dermatology. Based on the clinical assumption of pyoderma gangrenosum the patient received treatment with corticosteroids. The lesion proved unresponsive to the treatment and punch biopsies, of the affected area, were performed (Fig. 1A). Histopathological findings showed granuloma formation and CL was suggested as a possible cause. Amplification of Leishmania genomic sequences confirmed the diagnosis in August 2013. The patient was referred to the Department of Infectious Diseases, where treatment consisted of miltefosine 50 mg b.d. (twice daily) for 28 days. Post treatment, clinical examination showed regression of the cutaneous lesion, but not complete healing (Fig. 1B.)
Three months after the initial regression, the ulcer recurred. New biopsies sent for PCR showed Leishmania, leading to the conclusion of a treatment failure. Liposomal amphotericin B (1-AmB) $200 \mathrm{mg}$ IV daily was given for 10 days in March 2014.

After the infusions the lesion partly healed, but relapsed as treatment with $1-A m B$ ended (Fig. 2A). It was speculated that ADA treatment could be responsible for the relapse of CL. ADA treatment was therefore paused and a second course of $1-A m B$ was given. 16 doses of $1-\mathrm{AmB} 200 \mathrm{mg}$ were administered with 48 hours interval. Following this the patient was seen at the Department of Infectious Diseases and it was concluded that treatment, after pausing ADA, had been successful (Fig. 2B). The pausing of ADA was well tolerated for the duration of the CL treatment.
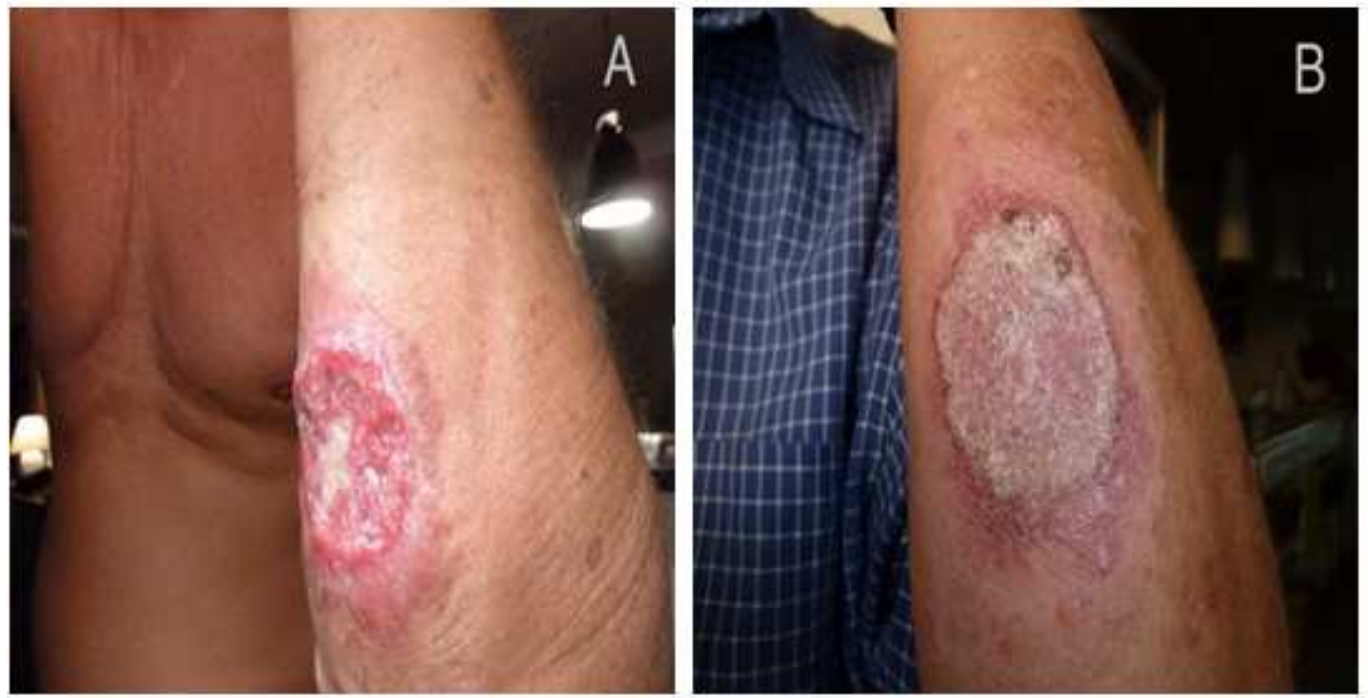

Fig. 1. Lesion before (A) and after miltefosin treatment (B)
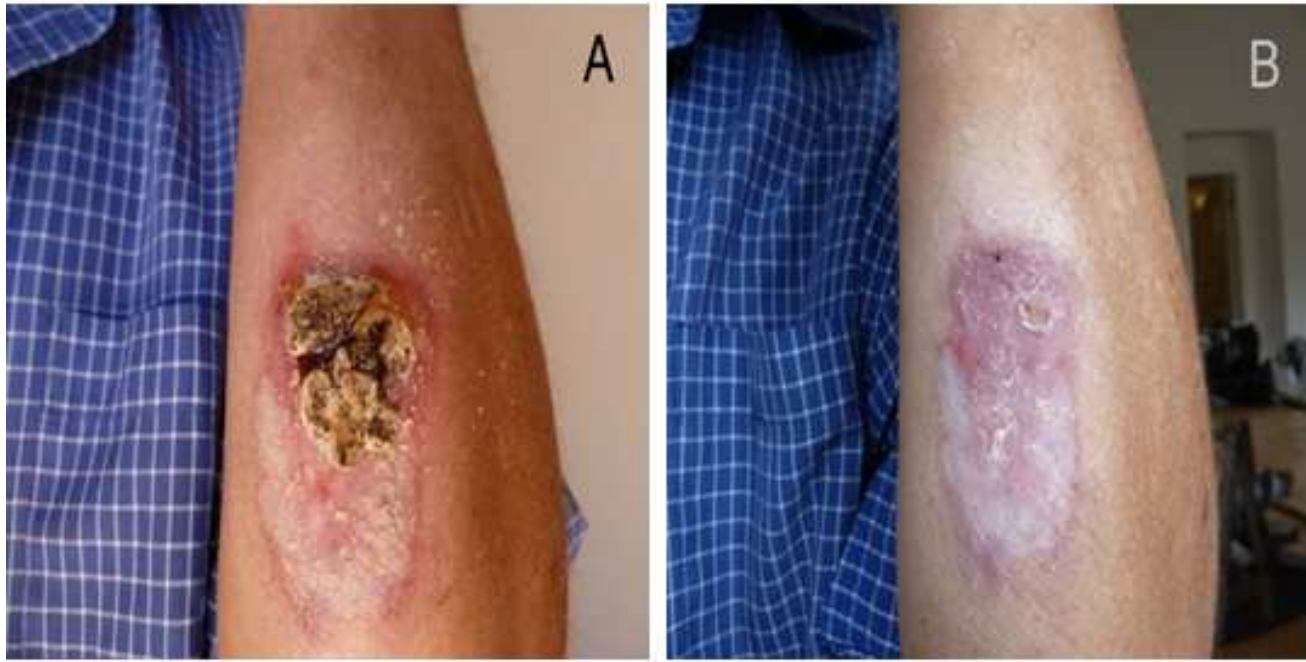

Fig. 2. Lesion before (A) and after second l-AmB treatment (B) 


\section{Discussion}

In Southern Europe leishmaniasis is solely caused by the subtype $L$. infantum and in Portugal domestic dogs present a large zoonotic reservoir. L. infantum can cause CL, although visceral leishmaniasis is the most common presentation of the disease (Campino et al., 2006). The protozoon is an obligate intracellular parasite, infecting macrophages, which are essential in the formation of granulomas. TNF- $\alpha$ antagonists are potent inhibitors of cytokines that play an important role in the formation of granulomas and the host's defense against intracellular infections such as tuberculosis and legionellosis. Studies of mice have shown that low levels of TNF- $\alpha$ and TNF- $\alpha$ antagonist treatment corresponds with larger, hyperparasited lesions less responsive to treatment (Titus et al., 1989; Murray et al., 2000). Data for drug efficiency in relation to treatment of $\mathrm{CL}$ is limited. Although literature is limited, $1-\mathrm{AmB}$ in the given dose has been shown to be effective in $97 \%$ of treated patients in one smaller study (Solomon et al., 2013). L-AmB is as polyene antibiotic with a specificity for macrophages due to its liposomal form. It targets ergosterol and causes death to the parasite by increased membrane permeability. Previously cases of $\mathrm{CL}$ in patients undergoing ADA treatment have been reported in literature (Gomes et al., 2012; Franklin et al., 2009). Gomes et al. (2012) reported a case of cutaneous leishmaniasis which responded to Glucantime treatment. In this case the authors found that there was no reason to believe that an association was present as the patient neither presented with disseminated or relapsing Leishmania. ADA was paused for the duration of the treatment. Franklin et al. (2009) described the case of a latent leishmania infection presumably activated by ADA treatment. The lesion responded to $1-A m B$ treatment.

Khan et al. (2010) described a case of disseminated $\mathrm{CL}$, in a patient treated with ADA for psoriatic arthritis. The patient responded to treatment with sodium stilbogluconate and $1-A m B$ treatment, but the patients underlying disease flared when ADA treatment was paused and ADA treatment was restarted under close monitoring. Zanger et al. (2012) found that numbers of case reports linking leishmania and anti TNF- $\alpha$ therapy were increasing. Looking at previous cases they found evidence that suggested that ertacenept treatment possibly should be favoured over ADA and infliximab treatment, in patients at risk of leishmania exposure.

\section{Conclusion}

We believe that repeated treatment failure of CL in this case was explained by the use of TNF- $\alpha$ antagonists.
Our case proved unresponsive to treatment with miltefosine and 1-AmB. Only after withdrawal of ADA did a second treatment course with $1-A m B$ result in healing. The use of TNF- $\alpha$ antagonists increases globally. This in turn requires increased focus on possible opportunistic infections and alterations of treatment regimes in relation to these. The growing consensus that ADA treatment should be paused for the duration of CL treatment is underlined by this case-report. In light of this case we find it recommendable that pausing TNF- $\alpha$ antagonist treatment should be considered in patients undergoing treatment for CL and that patients, following treatment, should be monitored closely, as small numbers of viable parasites in lesions may result in reactivation of the disease when ADA treatment is restarted. Furthermore it might be considered altering the treatment in favor of ertacenept in patients returning to, or living in, areas of endemic leishmaniasis.

\section{Conflicts of Interest}

The authors declare that they have no conflicts of interest.

\section{Author's Contributions}

Erik Otte: Wrote the paper, performed the litterature search.

Mikael Christiansen: Managed the humira treatment, commented on the manuscript.

Eskild Petersen: Managed the leishmania treatment, supervised writing of the manuscript, discussed the publication with the patient.

\section{Ethics}

We obtained permission from the patient to publish his data and the patient provided the phot's.

\section{References}

Alvar, J., I.D. Vélez, C. Bern, M. Herrero and P. Desjeux et al., 2012. Leishmaniasis worldwide and global estimates of its incidence. PLoS ONE. DOI: 10.1371 /journal.pone.0035671

Aspöck, H., T. Gerersdorfer, H. Formayer and J.Walochnik, 2008. Sandflies and sandfly-borne infections of humans in Central Europe in the light of climate change. Wien. Klin. Wochenschr, 120: 24-29. DOI: 10.1007/s00508-008-1072-8

Braun, J., R. van den Berg, X. Baraliakos, H. Boehm and R. Burgos-Vargas et al., 2011. 2010 Update of the ASAS/EULAR recommendations for the management of ankylosing spondylitis. Ann. Rheum. Dis., 70: 896-904. DOI: 10.1136/ard.2011.151027 
Campino, L., F. Pratlong, P. Abranches, J. Rioux and G. Santos-Gomes et al., 2006. Leishmaniasis in portugal: Enzyme polymorphism of Leishmania infantum based on the identification of 213 strains. Trop. Med. Int. Health, 11: 1708-1714. PMID: 17054751

Faber, W.R., R. Hoekzema, A. Bart, J. Zeegelaar and Henry J.C. de Vries, 2012. Cutaneous leishmaniasis acquired in Jura, France. Emerg. Infect. Dis., 18: 183-184. DOI: $10.3201 /$ eid1801.110408

Franklin, G., J. Greenspan and S. Chen, 2009. Antitumor necrosis factor-alpha therapy provokes latent leishmaniasis in a patient with rheumatoid arthritis. Ann. Clin. Lab Sci., 39: 192-195. PMID: 19429808

Gomes, K.W.P., A.N. Benevides, F.J.F. Vieira and M.P.D.M. Burlamaqui, 2012. Cutaneous leishmaniasis in a patient with ankylosing spondylitis using adalimumab. Rev. Bras. Reumatol., 52: 447-452. PMID: 22641598

Hodiamont, C.J., P. Kager, A. Bart and H.J.C. de Vries, 2014. Species-directed therapy for leishmaniasis in returning travellers: A comprehensive guide. PLoS Neglected Tropical Dis., DOI: 10.1371/journal.pntd.0002832

Khan, A., G. Coakley, C. Cosgrove and D. Lockwood, 2010. Let off the leash: Kala-azar following the use of tumour necrosis factor antibodies. BMJ Case Rep. DOI: 10.1136/bcr.04.2010.2878
Murray, H.W., A. Jungbluth, E. Ritter, C. Montelibano and M.W. Marino, 2000. Visceral leishmaniasis in mice devoid of tumor necrosis factor and response to treatment. Infect. Immun., 68: 6289-6293. PMID: 11035737

Solomon, M., F. Pavlotzky, A. Barzilai and E. Schwartz, 2013. Liposomal amphotericin B in comparison to sodium stibogluconate for Leishmania braziliensis cutaneous leishmaniasis in travelers. J. Am. Acad. Dermatol., 68: 284-289. DOI: $10.1016 /$ j.jaad.2012.06.014

Titus, R., G. Sherry and A. Cerami, 1989. Tumor necrosis factor plays a protective role in experimental murine cutaneous leishmaniasis. J. Exp. Med., 170: 2097-2104. PMID: 2584936

Van der Heijde, D., A. Kivitz, M.H. Schiff, J. Sieper and B.A. Dijkmans et al., 2006. Efficacy and safety of adalimumab in patients with ankylosing spondylitis: Results of a multicenter, randomized, double-blind, placebo-controlled trial. Arthritis Rheum., 54: 2136-2146. PMID: 16802350

Zanger, P., I. Kötter, P.G. Kremsner and S. Gabrysch, 2012. Tumor necrosis factor alpha antagonist drugs and leishmaniasis in Europe. Clin Microbiol. Infect., 18: 670-676. DOI: 10.1111/j.1469-0691.2011.03674 